\title{
Development and implementation of an international proficiency testing program for a neutralizing antibody assay for HIV-1 in TZM-bl cells
}

\author{
Christopher A. Todd ${ }^{\mathrm{a}, 1}$, Kelli M. Greene ${ }^{\mathrm{a}, 1}$, Xuesong Yu ${ }^{\mathrm{b}}$, Daniel A. Ozaki ${ }^{\mathrm{a}}$, Hongmei Gao ${ }^{\mathrm{a}}$, \\ Yunda Huang $^{\mathrm{b}}$, Maggie Wang ${ }^{\mathrm{b}}$, Gary $\mathrm{Li}^{\mathrm{c}}$, Ronald Brown ${ }^{\mathrm{c}}$, Blake Wood ${ }^{\mathrm{b}}$, M. Patricia \\ D'Souza $^{d}$, Peter Gilbert ${ }^{b}$, David C. Montefioria ${ }^{a}$, and Marcella Sarzotti-Kelsoe ${ }^{a},{ }^{*}$
}

Christopher A. Todd: chris.todd@duke.edu; Kelli M. Greene: kelli.greene@duke.edu; Xuesong Yu: xyu@scharp.org; Daniel A. Ozaki: daniel.ozaki@dm.duke.edu; Hongmei Gao: hongmei.gao@duke.edu; Yunda Huang: yunda@scharp.org; Maggie Wang: maggie@fhcrc.org; Gary Li: lig@qualitybiological.com; Ronald Brown: brownr@qualitybiological.com; Blake Wood: geebeewood@gmail.com; M. Patricia D’Souza: pdsouza@niaid.nih.gov; Peter Gillbert: pgilbert@scharp.org; David C. Montefiori: monte@duke.edu; Marcella Sarzotti-Kelsoe: marcella.sarzottikelsoe@dm.duke.edu

aDuke University Medical Center, Durham, NC, USA

bStatistical Center for HIV/AIDS Research and Prevention, Fred Hutchinson Cancer Research Center, Seattle, WA, USA

${ }^{\circ}$ Quality Biological, Inc., Gaithersburg, MD, USA

dNational Institutes of Health, National Institute of Allergy and Infectious Diseases, Division of AIDS, Bethesda, MD, USA

\section{Abstract}

Recent advances in assay technology have led to major improvements in how HIV-1 neutralizing antibodies are measured. A luciferase reporter gene assay performed in TZM-bl (JC53bl-13) cells has been optimized and validated. Because this assay has been adopted by multiple laboratories worldwide, an external proficiency testing program was developed to ensure data equivalency across laboratories performing this neutralizing antibody assay for HIV/AIDS vaccine clinical trials.

The program was optimized by conducting three independent rounds of testing, with an increased level of stringency from the first to third round. Results from the participating domestic and international laboratories improved each round as factors that contributed to inter-assay variability were identified and minimized. Key contributors to increased agreement were experience among laboratories and standardization of reagents.

A statistical qualification rule was developed using a simulation procedure based on the three optimization rounds of testing, where a laboratory qualifies if at least 25 of the 30 ID50 values lie within the acceptance ranges. This ensures no more than a $20 \%$ risk that a participating laboratory fails to qualify when it should, as defined by the simulation procedure. Five experienced reference laboratories were identified and tested a series of standardized reagents to derive the acceptance ranges for pass-fail criteria. This Standardized Proficiency Testing Program is the first available for the evaluation and documentation of assay equivalency for laboratories performing HIV-1 neutralizing antibody assays and may provide guidance for the development of future proficiency testing programs for other assay platforms.

\section{(C) 2011 Elsevier B.V. All rights reserved.}

*Corresponding author at: 2812 Erwin Road, Ste. 301, Erwin Terrace II, Duke University Medical Center, Durham, NC 27705, USA. Tel.: +19196846373; fax: +19196817766.

1 These authors contributed equally to this paper. 


\section{Keywords}

Neutralizing; Antibody; Assay; Proficiency; HIV; TZM-bl

\section{Introduction}

As the spread of HIV/AIDS increases worldwide, a safe and efficacious vaccine remains the cornerstone for a prevention strategy to ending the HIV-1 epidemic. The consensus view is that an effective vaccine will need to elicit coordinated B cell, CD4+ and CD8+ T cell responses. Neutralizing antibodies are correlates of protective immunity of most FDAapproved vaccines against infectious agents; consequently, standardized assessments of the neutralizing antibody response are a critical component of the HIV-1 vaccine discovery process (D'Souza et al., 1997), where large numbers of samples are assayed against multiple strains of the virus in different laboratories. A variety of assays have been developed in laboratories worldwide to test specimens from HIV vaccine trial participants and from HIVinfected individuals for the presence of neutralizing antibodies against HIV-1. However, the results from these assays have been difficult to compare. A key aspect of creating a Proficiency Testing Program is to be able to achieve and document equivalent assay performance across multiple laboratories. A comparative study conducted by NeutNet described results generated in 15 laboratories using 16 different HIV-1 neutralization assays (Fenyo et al., 2009). Results from the NeutNet project demonstrated clear differences in assay sensitivity that were dependent on both the neutralizing reagent and the virus, and concluded that no single assay could be recommended for use alone as a potential correlate of vaccine efficacy (Fenyo et al., 2009).

Although a discussion on the validity of one assay versus another is beyond the scope of the present manuscript, of the 16 neutralization assays used by NeutNet, the TZM-bl assay proved to be the most capable of providing consistent measurements. The TZM-bl assay uses a stable cell line (TZM-bl, also called JC53bl-13) and a Tat-regulated firefly luciferase (Luc) reporter gene that is induced after infection with most strains of HIV-1 (Platt et al., 1998; Wei et al., 2002, 2003; Li et al., 2005, 2006). This cell line permits rapid, sensitive and reproducible measurements of neutralization after a single round of infection in a high throughput assay format (Wei et al., 2003; Montefiori, 2005). Moreover, this assay may be used with molecularly cloned, Env-pseudotyped viruses for greater reagent stability and traceability (Wei et al., 2003; Li et al., 2005, 2006). This luciferase reporter gene assay performed in TZM-bl cells (Montefiori, 2005) was optimized and validated by the Laboratory for AIDS Vaccine Research and Development at Duke University and the National Institute of Allergy and Infectious Diseases (NIAID) Vaccine Immunogenicity Tcell and Antibody Laboratory (NVITAL) associated with the Vaccine Research Center.

In addition to a high level of sensitivity with Env-pseudotyped viruses, the TZM-bl cell assay has a distinct advantage over traditional neutralizing antibody assays (e.g., PBMC assay), since utilization of a cell line as the assay target cell eliminates issues of donor to donor variability present when utilizing peripheral blood mononuclear cells (PBMC) (Montefiori, 2005). A possible limitation of the TZM-bl assay compared to the PBMC assay, is the higher levels of CD4 and CCR5 on TZM-bl cells, which may reduce the sensitivity of the assay for certain monoclonal antibodies (mAbs) (Choudhry et al., 2006). Although TZM-bl is not a natural target for infection, the results are often in line with those obtained in PBMC assays (Fenyo et al., 2009). Moreover, measurements of neutralization in the TZM-bl cell assay are not susceptible to the effects of endotoxin contamination of samples, as is the case for the PBMC assay (Geonnotti et al., 2010). The TZM-bl cell assay is more suitable for assay technology transfer, implementation and standardization in 
laboratories across the world due to these advantages. Since 2002, the National Institutes of Health-AIDS Research and Reference Reagent Program (NIH-ARRRP) have distributed over 720 vials of TZM-bl cells throughout the world (NIH-ARRRP Personal Communication). Because this assay has been adopted by many laboratories, an external proficiency testing procedure is needed to assure that multiple laboratories participating in the evaluation of HIV-1 candidate vaccines are producing equivalent assay results.

Here we describe the establishment and implementation of the first standardized proficiency testing program for laboratories using the TZM-bl assay for HIV-1 neutralizing antibodies. This process required three rounds of optimization and the design of a statistical rule for the establishment of acceptance criteria. The standardized program is supported by the National Institutes of Health (NIH), NIAID, Division of AIDS (DAIDS) and the Collaboration for AIDS Vaccine Discovery (CAVD) through a grant to the Comprehensive Antibody-Vaccine Immune Monitoring Consortium (CA-VIMC).

\section{Materials and methods}

\subsection{Participating laboratories}

The following laboratories participated in the initial development and/or formal implementation of the Proficiency Testing Program for the Neutralizing Antibody Assay for HIV-1 in TZM-bl Cells: Duke University Medical Center, Durham, NC, USA, (D. Montefiori, B. Haynes and H. Liao); Vaccine Research Center (VRC), Bethesda, MD, USA (J. Mascola); University of Alabama at Birmingham (UAB), Birmingham, AL, USA, (G. Shaw); Beth Israel Deaconess Medical Center (BIDMC), Boston, MA, USA, (M. Seaman); National Institute for Communicable Diseases (NICD), Johannesburg, South Africa, (L. Morris); Seattle Biomedical Research Institute, Seattle, WA, USA, (N. Doria-Rose and L. Stamatatos); Chiron Corporation, Emeryville, CA, USA, (S. Barnett); Walter Reed Army Institute of Research, Rockville, MD, USA, (V. Polonis); Istituto Superiore di Sanità, Rome, Italy, (F. Ferrantelli); Torrey Pines Institute for Molecular Studies, San Diego, CA, USA (J. Binley); Biomedical Primate Research Centre, Rijswijk, The Netherlands, (J. Heeney); Centers for Disease Control and Prevention, Atlanta, GA, USA, (D. Ellenberger); Uniformed Services University of the Health Sciences, Bethesda, MD, USA, (G. Quinnan); Joan and Sanford I. Weill Medical College of Cornell University, New York, NY, USA, (J. Moore and F. Schader); Quality Biological, Inc. (QBI), Gaithersburg, MD, USA, (R. Brown); Institut National de la Santé et de la Recherche Médicale (INSERM), Strasbourg, France, (C. Moog); University of Pittsburgh School of Medicine, Pittsburgh, PA, USA, (K. Stefano Cole); St. Jude Children's Research Hospital, Memphis, TN, USA, (J. Hurwitz); NIAID Vaccine Immune T-Cell and Antibody Laboratory (NVITAL), Gaithersburg, MD, USA, (R. Bailer); National AIDS Research Institute (NARI), Pune, India, (R. Paranjape); Makerere University-Walter Reed Project (MUWRP), Kampala, Uganda, (F. WabwireMangen); Armed Forces Research Institute of Medical Sciences (AFRIMS), Bangkok, Thailand, (M. de Souza); Siriraj Hospital, Bangkok, Thailand, (R. Sutthent); Y.R. Gaitonde Centre for AIDS Research and Education (YRG CARE), Chennai, India, (P. Balakrishnan); National Center for AIDS/STD Control and Prevention - China CDC (NCAIDS), Beijing, China, (Y. Shao); Fraunhofer Institut für Biomedizinische Technik (IBMT), Sulzbach, Germany (H. von Briesen), Fred Hutchinson Cancer Research Center (FHCRC), Seattle, WA (J. Overbaugh). Overall, 21 of these laboratories participated in the three optimization rounds of program development. Once the program formally commenced, 5 of the 21 laboratories and an additional 9 laboratories enrolled in the program. 


\subsection{Neutralizing antibody assay for HIV-1 in TZM-bl cells}

Neutralization was measured as a reduction in firefly luciferase (Luc) reporter gene expression in the presence of test sample compared to the absence of sample after a single round of infection in TZM-bl cells. TZM-bl (JC53-bl) cells were obtained from the NIH ARRRP (Cat.no. 8129) as contributed by John Kappes and Xiaoyun Wu (Derdeyn et al., 2000; Takeuchi et al., 2008). The cell line is a genetically engineered HeLa cell clone that expresses CD4, CXCR4 and CCR5 and contains Tat-responsive reporter genes for firefly luciferase (Luc) and E. coli $\beta$-galactosidase under regulatory control of an HIV-1 LTR (Platt et al., 1998; Wei et al., 2002). The cell line was maintained in Dulbecco's Modified Eagle Medium (DMEM) containing 10\% Fetal Bovine Serum (FBS), gentamicin or PenicillinStreptomycin and optional use of HEPES (4-(2-hydroxyethyl)-1-piperazineethanesulfonic acid). Cultures were incubated at $37{ }^{\circ} \mathrm{C}$ in a humidified $5 \% \mathrm{CO}_{2} / 95 \%$ air environment. Cell monolayers were split upon confluency by treatment with $0.25 \%$ Trypsin, 1 mM EDTA (Ethylenediaminetetraacetic acid).

The TZM-bl neutralization assay described here is a modified version of the assay described previously (Li et al., 2005; Montefiori, 2005). Serologic reagents to be tested for neutralizing activity were serially diluted in 96-well flat-bottom culture plates containing growth medium, followed by the addition of Env-pseudotyped virus that was previously titrated for optimal infectivity. Freshly trypsinized TZM-bl cells, containing an optimal concentration of DEAE-Dextran (as determined in each laboratory), were added to each well following a 30-90 min incubation period (varied between laboratories). The use of DEAEDextran was optional. Following a 24-72 h incubation period (varied between laboratories), culture medium was removed from each well and replaced with a luciferase reporter gene assay system reagent (Britelite, PerkinElmer or Brite-Glo, Promega). After a short incubation (minimum of $2 \mathrm{~min}$ ), lysates were transferred to 96-well plates for measurement of luminescence in a luminometer (various models were used: PerkinElmer, Berthold, TECAN, ThermoLabsystems, Molecular Devices, Millipore). The 50\% (80\%) inhibitory dose (ID50) (ID80) was defined as the reciprocal of the serologic reagent dilution that caused a 50\% (80\%) reduction in relative luminescence units (RLU) compared to virus control wells after subtraction of background RLU.

\subsection{Pseudovirus preparation and titration}

The Env-pseudotyped viruses used in all phases of the program included the subtype B reference strains AC10.0.29, CAAN5342.A2, PVO.4, QH0692.42, THRO4156.18, and WITO4160.33. All six Env-expressing plasmids are available from the NIH AIDS Research and Reference Reagent Program (ARRRP catalog numbers 11024, 11038, 11022, 11018, 11037, and 11033, respectively). 293T/17 Cells were obtained from the American Type Culture Collection (Cat.no. 11268). The cell line was maintained in DMEM containing 10\% Fetal Bovine Serum (FBS), gentamicin and HEPES. Cultures were incubated at $37{ }^{\circ} \mathrm{C}$ in a humidified $5 \% \mathrm{CO}_{2} / 95 \%$ air environment. The density of cell monolayers was reduced upon confluency by treatment with $0.25 \%$ Trypsin, $1 \mathrm{mM}$ EDTA and transfer of an aliquot of cells to new culture flasks.

During the first optimization round, laboratories prepared their own stocks of pseudovirus using the supplied plasmids. Methods for transfection included Fugene, Polyfect, Calcium Phosphate, GeneJuice, Lipofectamine, and LT1. In the subsequent rounds, pseudoviruses were prepared centrally by using a modified version of a procedure described previously ( $\mathrm{Li}$ et al., 2005). Briefly, exponentially dividing 293T/17 cells were co-transfected with an envexpressing plasmid and an env-deficient HIV-1 backbone vector pSG3 $\triangle \mathrm{Env}$ (NIH-ARRRP Cat.no. 11051), using the transfection reagent Fugene (Roche). Pseudovirus-containing culture supernatants were harvested two days after transfection, filtered $(0.45 \mu \mathrm{m})$, and 
stored at $-80^{\circ} \mathrm{C}$ in 1-ml aliquots. The optimal tissue culture infectious dose (TCID) of a single thawed aliquot of each batch of pseudovirus was determined in TZM-bl cells. Serial dilutions were performed in quadruplicate wells of clear, flat-bottom, 96-well culture plates for a total of 11 dilution steps. Pseudovirus was omitted from the last column of the 96-well plate (cell control). Freshly trypsinized cells containing an optimal concentration of DEAEDextran were added to each well, and the plates were incubated at $37{ }^{\circ} \mathrm{C}$ in a humidified $5 \%$ $\mathrm{CO}_{2} / 95 \%$ air environment. Following the 48-hour incubation, culture medium was removed from each well and a luciferase reporter gene assay system reagent was added to each well. After cell lysis, lysate was transferred to solid black 96-well plates for measurements of luminescence.

\subsection{Serologic reagents}

The serologic reagents tested in the first three optimization rounds included HIV-positive plasma pool 1 (subtype C) and pool 2 (subtype B), mAbs (IgG1b12, 2F5, 4E10, 2G12, TriMab), a recombinant protein (sCD4), and a HIV-negative serum pool. The clade C plasma pool was prepared from six to ten subjects with pure clade classifications from anonymous, HIV-positive blood bank units collected in Tanzania (Li et al., 2006). The U.S. HIV-1-positive pool B was prepared from plasma samples obtained from subjects participating in a vaccine trial of recombinant gp160 (Walter Reed HSRRB lot no. A5392). Both plasma pools were kindly provided by V. Polonis, Walter Reed Army Institute of Research. A normal serum pool was prepared from leukopaks from four HIV-1-negative subjects (BRT Laboratories, Inc., Baltimore, MD). Human mAb IgG1b12 was provided by the QBI and the Vaccine Research Program, Division of AIDS, NIAID, NIH. Human mAbs 2G12, 2F5, and 4E10 were purchased from Polymun, Inc. (Vienna, Austria). TriMab is a mixture of three $\mathrm{mAbs}(\mathrm{IgG1b} 12,2 \mathrm{G} 12$, and 2F5) prepared as a 1-mg/ml stock solution containing $333 \mu \mathrm{g}$ of each $\mathrm{mAb} / \mathrm{ml}$ in phosphate-buffered saline. Recombinant sCD4 comprising the full-length extracellular domain of human CD4 produced in Chinese hamster ovary cells was obtained from Progenics Pharmaceuticals, Inc. (Tarrytown, NY). The serologic reagents used in the ongoing external Proficiency Testing Program, to which the laboratories are blinded, are comprised of some of the reagents used in the first three optimization rounds of testing.

\subsection{Data reporting and statistical analysis}

During the three rounds of optimization, participating laboratories reported both ID50 and ID80 values electronically by completing and submitting a data entry template to the Statistical Center for HIV/AIDS Research and Prevention (SCHARP). Each participating laboratory received and submitted an assay checklist for collection of assay parameter information. This checklist was used to compare assay methods and examine potential causes of inter-laboratory assay variation. Confidentiality of results was mandated across participating laboratories throughout all rounds of testing.

All statistical analyses were based on $\log 10$-transformed values of ID50 and ID80 due to skewness of the ID values. For each round, the mean, standard deviation and coefficient of variation ( $\mathrm{CV}=$ =standard deviation/mean) of the ID50 and ID80 values for each serologic reagent-virus combination were calculated for each participating laboratory. The CVs were used to assess overall variability across laboratories. After examining the data from all three optimization rounds, the program decided not to use ID80 titers as criteria for acceptance due to high percentage of ID80 titers below the assay detection limit. The equivalency rate between any two participating laboratories (i.e., laboratory A versus laboratory B) was calculated as the percentage of ID50 values that agreed within three fold of each other, i.e., $\mid \log _{10}$ (Lab A ID50)- $\log _{10}$ (Lab B ID50) $I \leq \log _{10}$. Laboratory pairs were considered equivalent when $\geq 80 \%$ of the paired ID50 titers were within 3 -fold of each other. 


\subsection{Study design}

A flow chart illustrating the study design is shown in Fig. 1. Proficiency test kits containing serologic reagents and pseudoviruses were assembled and distributed by Quality Biological, Inc. (Gaithersburg, MD) for all phases of the program. Pseudoviruses included in proficiency testing kits were produced in a GCLP-compliant manner by the CAVD/CAVIMC Central Reference Laboratory (CRL) at Duke University (Laboratory for AIDS Research and Development) and the Fraunhofer IBMT in Germany. The GCLP-compliant environment included Standard Operating Procedures for pseudovirus preparation and titration, serologic reagent testing using a validated TZM-bl assay, and the use of calibrated and validated equipment by trained and competent operators under the oversight of a Quality Assurance Unit. The Proficiency Testing Program was developed in two phases.

In phase I, three optimization rounds were conducted over a four year period involving domestic (US) and international laboratories to develop and validate a method for measuring inter-laboratory equivalency. The first round of optimization included 13 laboratories located in the United States and four international sites. Each laboratory received a kit containing eight blinded serologic reagents, selected for their low, medium and high neutralization phenotypes. The individual laboratories were responsible for acquiring the six Env-expressing plasmids and the backbone plasmid and using these independently to produce their own pseudovirus stocks. All participating laboratories received reference protocols for "Pseudovirus Preparation and Titration" and for "Neutralizing Antibody Assay for HIV-1 in TZM-bl Cells"; however, each site was allowed to use its own site-specific protocol. The goal of the first round of optimization was to examine the equivalence of the results across participating laboratories under conditions of relaxed standardization.

The second round of optimization involved 15 participating laboratories, 3 of which were international. Each laboratory received a kit containing six centrally prepared pseudovirus stocks as well as ten blinded serologic reagents selected for their low, medium and high neutralization phenotypes, two of which were duplicates. The objective of the second round of testing was to confirm whether the major source of inter-laboratory assay variability observed in the first round of optimization could be attributed to differences in the neutralization properties of pseudovirus stocks produced in each laboratory. To eliminate this possible variable, each laboratory was required to use centrally prepared pseudoviruses at the recommended dilution provided with the test instructions.

The third round of optimization was conducted by six experienced laboratories, five located in the United States and one located at an international site. Experienced laboratories were defined as having performed $>1000$ neutralization assays in TZM-bl Cells. All six laboratories received identical proficiency test kits containing six centrally prepared stocks of pseudovirus and twelve blinded (six duplicates) serologic reagents selected for their low, medium and high neutralization phenotypes and were assayed in duplicate to assess intralaboratory variability. The primary goal of the third round of optimization was to establish criteria to qualify laboratories by assessing intra-laboratory and inter-laboratory variability among the experienced laboratories. Higher levels of stringency ( $\geq 90 \%$ equivalence) were applied to this round of testing. At the conclusion of phase I, a statistical qualification rule was developed to create acceptance ranges for the Standardized Proficiency Testing Program.

In phase II, the Standardized Proficiency Testing Program was implemented using the predefined qualification rule. Proficiency Test Kits $(\mathrm{n}=250)$ were assembled at QBI under GCLP conditions and monitored by the CAVD/CA-VIMC Central Quality Assurance Unit. The kits were stored in temperature-controlled $-80^{\circ} \mathrm{C}$ freezers and tested once a year by five reference laboratories to confirm the acceptance criteria over time. Each kit contained 
six pseudoviruses and five blinded serologic reagents selected for their low, medium and high neutralization phenotypes, for a total of 30 serologic reagent/virus combinations used previously in the definition of the statistical qualification rule. The five serologic reagents were contributed by NIAID/DAIDS and the CAVD. The six pseudoviruses included in the kits expressed the Envs of strains AC10.0.29, CAAN5342.A2, PVO.4, QH0692.42, THRO4156.18, and WITO4160.33. The panel of pseudoviruses and serologic reagents included in the kits represent a broad range of antigenic diversity and specificity (Li et al., 2005). Participating laboratories were given one month to conduct the neutralization assays, from receipt of the kit to upload of the results to the Atlas portal maintained by SCHARP.

Program enrollment began in August of 2009 for all laboratories performing Luc-based neutralization assays for HIV-1 in TZM-bl cells. Participating laboratories received a standardized proficiency test kit and instructions for kit analysis and data submission. Laboratories that passed the test were deemed proficient in TZM-bl assay performance for a period of six months, at which time the laboratory is requested to complete the test again. This Proficiency Testing Program is current and ongoing.

\section{Results}

\subsection{Proficiency testing program phase I ( 3 rounds of optimization testing)}

Table 1 illustrates the results from the first round of testing. The data points for each serologic reagent-virus combination were independently compared between two laboratories, and defined as laboratory paired ID50 titers, across all 17 participating laboratories. Laboratory pairs were considered equivalent when $\geq 80 \%$ of the paired ID50 were within 3-fold of each other. For the first round of optimization, 46 out of 171 (27\%) laboratory pairs generated ID50 titers within 3-fold of each other $\geq 80 \%$ of the time. Laboratories 16 and 17 each performed two independent proficiency tests: one using the reference protocol provided to all participating laboratories, and another using their sitespecific procedure. An acceptable level of equivalency was achieved in both laboratories using either protocol.

Table 2 shows results from the second round of optimization, for which centrally prepared pseudovirus stocks were provided to all laboratories. Results for each serologic reagent/virus combination were independently compared between two laboratories, and defined as laboratory paired ID50 titers, across all 15 participating laboratories. Of the 105 pairs of laboratories, 54 (51\%) had $\geq 80 \%$ ID50 titers within 3-fold of each other. The laboratories participating in round 2 were able to identify low, medium, and high ID50 titers (Fig. 2).

A third round of optimization was performed to identify high equivalency ( $\geq 90 \%)$ among experienced laboratories using more stringent criteria than in the previous two optimization rounds. Six laboratories were chosen to participate in the third round because they had completed $>1000$ neutralization assays in TZM-bl cells. Overall, the pair-wise equivalency rates were $>90 \%$ among laboratories $1,2,3,5$ and 6 . The equivalency rates between laboratory 4 and other laboratories were less than $90 \%$ (Table 3 ). For this reason, laboratory 4 was not included as one of the reference labs for the development of the statistical rule. For each ID50 value from the duplicate combinations, the average $\mathrm{CV}$ was calculated to examine intra-laboratory variability. The range of $\mathrm{CVs}$ for the laboratories was 0.069 to 0.176 (data not shown). As in round 2, the five equivalent laboratories were able to detect low, medium, and high ID50 values. In addition, ID50 values captured in the third round were less variable than in the second round (Table 5 and Fig. 2). 


\subsection{Developing the statistical rule}

A statistical qualification rule was developed based on the data from five equivalent laboratories identified in the third round of optimization testing. The objective was to determine whether two vaccines are different in terms of generating neutralizing antibody responses, above and beyond any potential systematic error that occurs from using two separate laboratories to perform the assays. Based on the qualification rule, we have assurance that titers for the new laboratory may have up to a 2-fold systematic error compared to values from the reference laboratories. A 2-fold systematic difference in ID50 values was set such that results of two vaccine trials, each with 30-50 volunteers, could be compared, by qualified laboratories, with adequate statistical power to detect moderate differences in magnitude and breadth of the neutralizing antibody response (e.g., can detect 5 -fold differences in mean area under the magnitude and breadth (AUC-MB) curves).

Using the statistical qualification rule defined above, the pre-specified acceptance ranges were calculated for the first lot of 250 proficiency testing kits for the external program using the ID50 values obtained from the five reference laboratories as identified in the third round of optimization testing. Each acceptance range was defined by a $90 \%$ prediction interval that is centered around the geometric mean of ID50 values from the five reference laboratories. The width of the interval is determined by an estimate of the standard deviation of the $\log 10$-transformed ID50s. The truncated mean and standard deviations were estimated by removing the two highest and two lowest results from the 15 results for each serologic reagent/pseudovirus combination (5 laboratories $\times 3$ test kits each). At least 3 repeats per reference laboratory were needed to estimate the standard deviation of ID50s for each combination. Given inputs of the 30 mean estimates and the 30 standard deviation estimates, a simulation procedure was used to select the cut-off integer 25 to ensure that there is no more than a $20 \%$ risk that participating laboratories fail to qualify when they should. A laboratory qualifies if at least 25 of the 30 ID50 values lie within the pre-specified acceptance ranges defined above, determined for each of the 30 serologic reagent-virus combinations. These acceptance intervals depend critically on the standard deviation estimates, and will change when a new lot of test-kits are developed in the future.

\subsection{Enrollment and results from the standardized proficiency testing program}

Following enrollment in the Standardized Proficiency Testing Program, 14 laboratories participated and successfully completed the assays for the formal testing as of June 2011. Some laboratories participated in the program multiple times since its inception. As an example, Fig. 3 provides the distribution of neutralization titers for the 14 participating laboratories for one of the five serologic reagents against the six pseudoviruses in each kit. Data from Fig. 4 show the distribution of scores from all proficiency test runs (83\% is the lower limit of acceptance).

\section{Discussion}

During the HIV vaccine discovery process, vaccine induced neutralizing antibody responses are measured at multiple time points and often in multiple laboratories. In order to accurately compare results across different laboratories, it is helpful to first assure the data equivalency of the laboratories that will be making the assessments. To facilitate this process, we have developed a standardized proficiency testing program for standardized measurements of HIV-1-specific neutralizing antibodies in the TZM-bl assay. Three rounds of optimization involving 21 different test laboratories were required to design the final proficiency testing kit and to develop a statistical rule and appropriate acceptance criteria for the proficiency testing program. The first round of optimization was conducted under conditions of relaxed standardization in order to determine the baseline inter-laboratory 
variability inherent in conducting assays in multiple laboratories utilizing various protocols and reagents. Overall, the results showed very little assay equivalency among laboratories, with only $27 \%$ of the laboratory pairs able to generate ID50 titers within 3-fold of each other $\geq 80 \%$ of the time. Specific information regarding the assay procedure used in each laboratory was collected prospectively and analyzed retrospectively to identify variables that might account for inconsistencies in assay results. Laboratories 16 and 17 utilized two SOPs (one provided with the kit and their site-specific SOP) to examine differences in neutralization results between the two protocols. The key differences were the concentration of DEAE-Dextran $(15 \mu \mathrm{g} / \mathrm{ml}$ vs. $37.5 \mu \mathrm{g} / \mathrm{ml})$ and $/$ or the omission of it at the time of infection of the cells. Additionally, the length of incubation time was varied between the protocols for the pseudovirus and serologic reagents. No differences were detected using the variations of the protocols (Table 1). A major variable that tracked with assay inconsistency was pseudovirus production, which was performed independently in each laboratory. Subsequently, ancillary experiments were conducted and showed that differences in the neutralization properties of the pseudovirus stocks generated in each laboratory could be a major source of assay variation in the first round of optimization.

In an effort to improve equivalency among laboratories, centrally prepared and titrated pseudovirus stocks were used in a second round of optimization. Duplicate reagents were used in the second optimization round to examine intra-laboratory variability. Results from round 2 exhibited a near 2-fold improvement over the first optimization round (Tables 1 and 2). In round $2,51 \%$ of the laboratory pairs were within 3 -fold of each other $\geq 80 \%$ of the time as compared to $27 \%$ in round 1 . The results suggested that the improvement can be attributed to the use of identical stocks of centrally prepared pseudovirus across all participating laboratories since the other conditions in the first round of optimization were not changed. In addition, laboratories with greater overall experience in performing the TZM-bl cell neutralization assay (>1000 assays) demonstrated higher levels of equivalency (data not shown).

The goal of the third round of optimization was to evaluate the reproducibility and consistency of equivalent results from the first two rounds of optimization, to develop a standard operating procedure for proficiency testing, and to identify equivalent laboratories under more stringent criteria ( $\geq 90 \%$ equivalency) among experienced laboratories. These laboratories would be used to establish the statistical rule necessary to develop the acceptance ranges. As shown in Fig. 2, significant differences were observed when comparing ID50 titers obtained from rounds 2 and 3 . Therefore, acceptance ranges would need to be determined for each new lot of kits. However, Table 5 illustrates that overall the $\mathrm{CV}$ decreased with each subsequent round of optimization. This is attributed to the utilization of highly experienced laboratories performing the assay in round 3 .

Results from the third round of optimization indicated that $\geq 90 \%$ equivalency could be achieved in five of the six laboratories (Table 3). Although all six laboratories exhibited intra-laboratory equivalency $(\mathrm{CV} \leq 0.2)$, one failed to demonstrate inter-laboratory equivalency and was therefore considered an outlier for the development of the statistical rule. These results suggest that intra-laboratory equivalency is not a predictor of interlaboratory equivalency. When evaluating vaccine-elicited antibody responses across laboratories, it is imperative that laboratories demonstrate proficiency in addition to intralaboratory competency.

Following the development of a statistical rule, the program was transitioned from optimization to a standardized proficiency testing program. Kits were developed and assembled under GCLP conditions using standardized reagents. These kits included the same panel of centrally prepared pseudoviruses previously utilized, as well as a set of 
serologic reagents capable of capturing low, medium, and high ID50 values. Proficiency testing kits were quality-controlled upon production and on an annual basis in the following years by testing in the reference laboratories. The acceptance criteria for each lot of kits were determined based on the assay results generated by the five reference laboratories assaying three kits each (Table 4). Data from this testing showed that there was high interand intra-laboratory equivalency ( $>90 \%)$. Proficiency kits will continue to be evaluated on an annual basis for reagent stability and confirmation of acceptance ranges.

Enrollment in the Standardized Proficiency Testing Program for the TZM-bl assay is open and free of charge to any NIAID and/or CAVD-sponsored laboratory performing the assay. Upon request for enrollment, participating laboratories receive a proficiency testing kit, along with testing and data reporting instructions. The proficiency testing guidelines request that test results be submitted to the web based Atlas Science Portal (http://atlas.scharp.org), supported by SCHARP, within one month of receipt of the kit. Upon data submission, test values are compared to established acceptance range for determination of equivalency. The Proficiency Testing Program Coordinator reports proficiency testing results to the participating laboratory along with an evaluation of performance. Should a laboratory fail the test, the Program Coordinator will assist in determining the root cause of the failure and recommend that the laboratory document any measures taken to prevent repeat occurrences. In addition, a new proficiency test kit will be forwarded to the laboratory for repeat testing after corrective actions have been implemented. All participant data remains highly confidential. Thus far, 14 laboratories have participated in the Standardized Proficiency Testing Program (many on multiple occasions). While every participating laboratory passed the tests, scores ranged from the lowest acceptable number of correct ID50 values (83\%) to the highest number (100\%).

Although proficiency testing programs for neutralizing antibody assays against other pathogens exist, this is the first program available for the evaluation and documentation of assay equivalency for laboratories measuring neutralizing antibody responses against HIV-1 (Poirier et al., 2005). This is an important program for those laboratories that operate in a GCLP environment. In compliance with GCLP, a proficiency testing program for an endpoint immunogenicity assay should be based on the detection and/or quantitation of specific analytes under predetermined laboratory procedures and should be provided on a routine basis (Sarzotti-Kelsoe et al., 2009). Results from participating laboratories should agree with statistically pre-established acceptance ranges. A reporting structure should exist to inform laboratories if their results are within the acceptable range. All of these GCLP criteria are satisfied in the Standardized Proficiency Testing Program for laboratories performing the TZM-bl neutralizing antibody assay. This proficiency testing program is a critical component for assuring the equivalency of evaluations of vaccine-elicited neutralizing antibody responses against HIV-1 in multiple laboratories. Moreover, it serves as a component of a qualification process for certifying laboratories to perform the TZM-bl neutralization assay in support of human clinical trials (D. Ozaki, manuscript in preparation). The Global HIV/AIDS Vaccine Enterprise (GHAVE) Scientific Strategic Plan stressed the importance of regional/satellite laboratories in conjunction with core/reference laboratories for both clinical and preclinical work. More recently, emphasis was placed on the broad dissemination of laboratory platforms to countries where trials are being conducted (Coordinating Committee of the Global HIV/AIDS Vaccine Enterprise, 2005; The Council of the Global HIV Vaccine Enterprise, 2010). Specifically, all laboratories involved in the evaluation of neutralizing antibody responses in a recently completed Phase IIb efficacy trial in Thailand (RV144) (Rerks-Ngarm et al., 2009) were required to successfully complete routine proficiency testing using the standardized proficiency testing program. The development of this program may provide guidance for the establishment of future proficiency testing programs for other assay platforms. 


\section{Conclusion}

Three rounds of testing in 21 different laboratories were required to develop the appropriate acceptance criteria for the proficiency test. Variability among laboratories decreased after each round. This can be attributed to the use of the same stocks of pseudovirus across laboratories. Use of a common SOP for this assay had no effect on variability; however, experience at running the assay did lead to more concordance between labs. The proficiency test panel used for the program is capable of capturing low, medium, and high ID50 values. The reference laboratories detected no significant kit-to-kit variation. This uniformity may be attributed to the fact that the kits were produced under GCLP conditions using highly standardized reagents. The TZM-bl cell assay is a reliable and validated method for evaluating vaccine-elicited neutralizing antibody responses against HIV-1.

\section{Acknowledgments}

This program is jointly supported by the Bill \& Melinda Gates Foundation's Collaboration for AIDS Vaccine Discovery/Comprehensive Antibody Vaccine Immune Monitoring Consortium, grant number 38619, Collaboration for AIDS Vaccine Discovery/Vaccine Immunology Statistical Center, grant number 38744, National Institutes of Health grant AI30034, and the NIH/NIAID Reagent Resource Support Program for AIDS Vaccine Development, Quality Biological, Inc., Gaithersburg, MD. This paper was submitted by one author in her capacity as an employee of NIH, but the views expressed in this paper do not necessarily represent those of NIH. We also thank all of the laboratories that participated in the development of this program.

\section{References}

Choudhry V, Zhang MY, Harris I, Sidorov IA, Vu B, Dimitrov AS, Fouts T, Dimitrov DS. Increased efficacy of HIV-1 neutralization by antibodies at low CCR5 surface concentration. Biochem Biophys Res Commun. 2006; 348:1107. [PubMed: 16904645]

Coordinating Committee of the Global HIV/AIDS Vaccine Enterprise. The Global HIV/AIDS Vaccine Enterprise: scientific strategic plan. PLoS Med. 2005; 2(2):e25.10.1371/journal.pmed.0020025 [PubMed: 15740411]

D’Souza MP, Livnat D, Bradac JA, Bridges SH. Evaluation of monoclonal antibodies to human immunodeficiency virus type 1 primary isolates by neutralization assays: performance criteria for selecting candidate antibodies for clinical trials. AIDS Clinical Trials Group Antibody Selection Working Group. J Infect Dis. 1997; 175:1056. [PubMed: 9129066]

Derdeyn CA, Decker JM, Sfakianos JN, Wu X, O'Brien WA, Ratner L, Kappes JC, Shaw GM, Hunter E. Sensitivity of human immuno-deficiency virus type 1 to the fusion inhibitor T-20 is modulated by coreceptor specificity defined by the V3 loop of gp120. J Virol. 2000; 74:8358. [PubMed: 10954535]

Fenyo EM, Heath A, Dispinseri S, Holmes H, Lusso P, Zolla-Pazner S, Donners H, Heyndrickx L, Alcami J, Bongertz V, Jassoy C, Malnati M, Montefiori D, Moog C, Morris L, Osmanov S, Polonis V, Sattentau Q, Schuitemaker H, Sutthent R, Wrin T, Scarlatti G. International network for comparison of HIV neutralization assays: the NeutNet report. PLoS One. 2009; 4:e4505. [PubMed: 19229336]

Geonnotti AR, Bilska M, Yuan X, Ochsenbauer C, Edmonds TG, Kappes JC, Liao HX, Haynes BF, Montefiori DC. Differential inhibition of human immunodeficiency virus type 1 in peripheral blood mononuclear cells and TZM-bl cells by endotoxin-mediated chemokine and gamma interferon production. AIDS Res Hum Retroviruses. 2010; 26:279-291. [PubMed: 20218881]

Li M, Gao F, Mascola JR, Stamatatos L, Polonis VR, Koutsoukos M, Voss G, Goepfert P, Gilbert P, Greene KM, Bilska M, Kothe DL, Salazar-Gonzalez JF, Wei X, Decker JM, Hahn BH, Montefiori DC. Human immunodeficiency virus type 1 env clones from acute and early subtype B infections for standardized assessments of vaccine-elicited neutralizing antibodies. J Virol. 2005; 79:10108. [PubMed: 16051804]

Li M, Salazar-Gonzalez JF, Derdeyn CA, Morris L, Williamson C, Robinson JE, Decker JM, Li Y, Salazar MG, Polonis VR, Mlisana K, Karim SA, Hong K, Greene KM, Bilska M, Zhou J, Allen S, Chomba E, Mulenga J, Vwalika C, Gao F, Zhang M, Korber BT, Hunter E, Hahn BH, Montefiori 
DC. Genetic and neutralization properties of subtype $C$ human immunodeficiency virus type 1 molecular env clones from acute and early heterosexually acquired infections in Southern Africa. J Virol. 2006; 80:11776. [PubMed: 16971434]

Montefiori DC. Evaluating neutralizing antibodies against HIV, SIV, and SHIV in luciferase reporter gene assays. Curr Protoc Immunol. 2005; Chapter 12:Unit 12.11. [PubMed: 18432938]

Platt EJ, Wehrly K, Kuhmann SE, Chesebro B, Kabat D. Effects of CCR5 and CD4 cell surface concentrations on infections by macrophagetropic isolates of human immunodeficiency virus type 1. J Virol. 1998; 72:2855. [PubMed: 9525605]

Poirier B, Leparc-Goffart I, Crance JM, Fleury H, Garin D, Gut JP, Tissier MH, Fuchs F. Establishment of a national network of validated and qualified laboratories for neutralizing antivaccinia antibodies titration. Biol J Int Assoc Biol Stand. 2005; 33:277.

Rerks-Ngarm S, Pitisuttithum P, Nitayaphan S, Kaewkungwal J, Chiu J, Paris R, Premsri N, Namwat C, de Souza M, Adams E, Benenson M, Gurunathan S, Tartaglia J, McNeil JG, Francis DP, Stablein D, Birx DL, Chunsuttiwat S, Khamboonruang C, Thongcharoen P, Robb ML, Michael NL, Kunasol P, Kim JH. Vaccination with ALVAC and AIDSVAX to prevent HIV-1 infection in Thailand. N Engl J Med. 2009; 361:2209. [PubMed: 19843557]

Sarzotti-Kelsoe M, Cox J, Cleland N, Denny T, Hural J, Needham L, Ozaki D, Rodriguez-Chavez IR, Stevens G, Stiles T, Tarragona-Fiol T, Simkins A. Evaluation and recommendations on good clinical laboratory practice guidelines for phase I-III clinical trials. PLoS Med. 2009; 6:e1000067. [PubMed: 19536325]

Takeuchi Y, McClure MO, Pizzato M. Identification of gammaretroviruses constitutively released from cell lines used for human immunodeficiency virus research. J Virol. 2008; 82:12585. [PubMed: 18842727]

The Council of the Global HIV Vaccine Enterprise. The 2010 scientific strategic plan of the Global HIV Vaccine Enterprise. Nat Med. 2010; 16:981. [PubMed: 20823883]

Wei X, Decker JM, Liu H, Zhang Z, Arani RB, Kilby JM, Saag MS, Wu X, Shaw GM, Kappes JC. Emergence of resistant human immunodeficiency virus type 1 in patients receiving fusion inhibitor (T-20) monotherapy. Antimicrob Agents Chemother. 2002; 46:1896. [PubMed: 12019106]

Wei X, Decker JM, Wang S, Hui H, Kappes JC, Wu X, Salazar-Gonzalez JF, Salazar MG, Kilby JM, Saag MS, Komarova NL, Nowak MA, Hahn BH, Kwong PD, Shaw GM. Antibody neutralization and escape by HIV-1. Nature. 2003; 422:307. [PubMed: 12646921] 


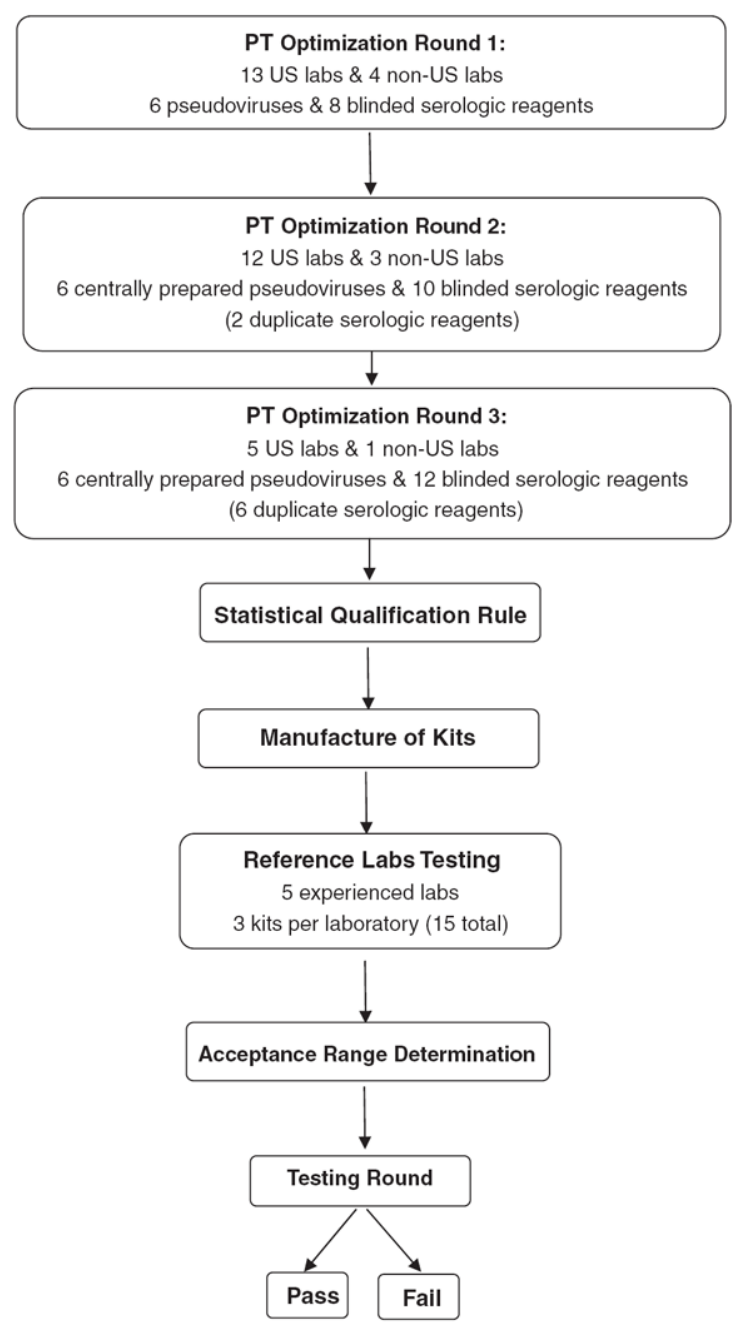

Fig. 1.

Study design for the proficiency testing program. This schematic flow chart illustrates all phases used in the development of the Standardized Proficiency Testing Program for measurements of HIV-1-specific neutralizing antibodies in the TZM-bl assay. 

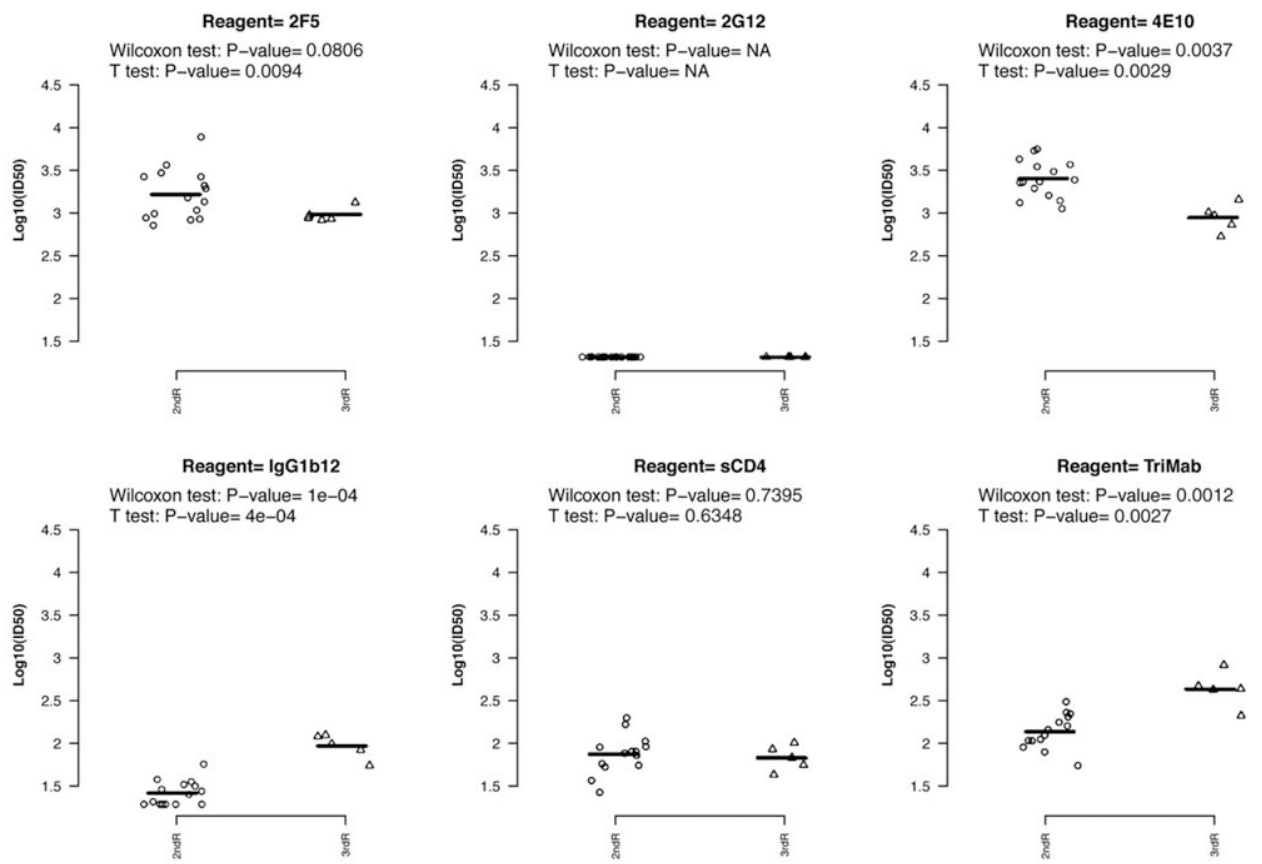

Fig. 2.

Comparison of harmonized means between the second and third rounds of optimization. Shown are Log10 Transformed ID50 values from each serologic reagent/pseudovirus combination from the second and third rounds of optimization. All of the antibodies were used at a starting concentration of $0.5 \mathrm{mg} / \mathrm{ml}$. Bars represent harmonized means of results for all participating laboratories. Differences between harmonized means were examined between the second and third rounds as indicated in each panel. 


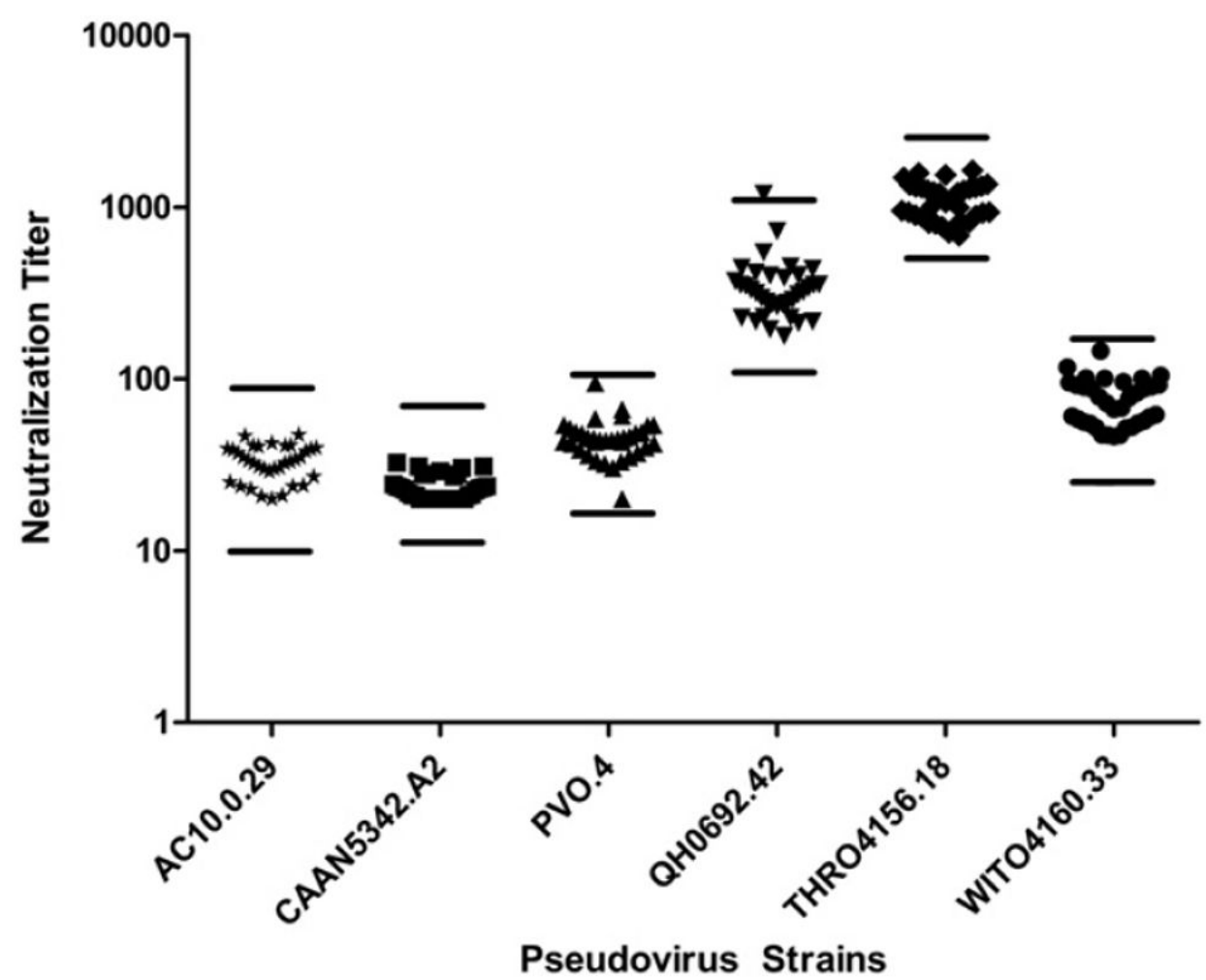

Fig. 3.

Example of neutralization titers for laboratories participating in the standardized proficiency testing program. Proficiency testing results illustrating the range of ID50 neutralization titers obtained in the participating laboratories when testing one serologic reagent against all six pseudovirus strains. Bars indicate the upper and lower limits of the acceptance ranges. Data points represent 37 individual test results from 14 participating laboratories from August 2009 to June 2011. 


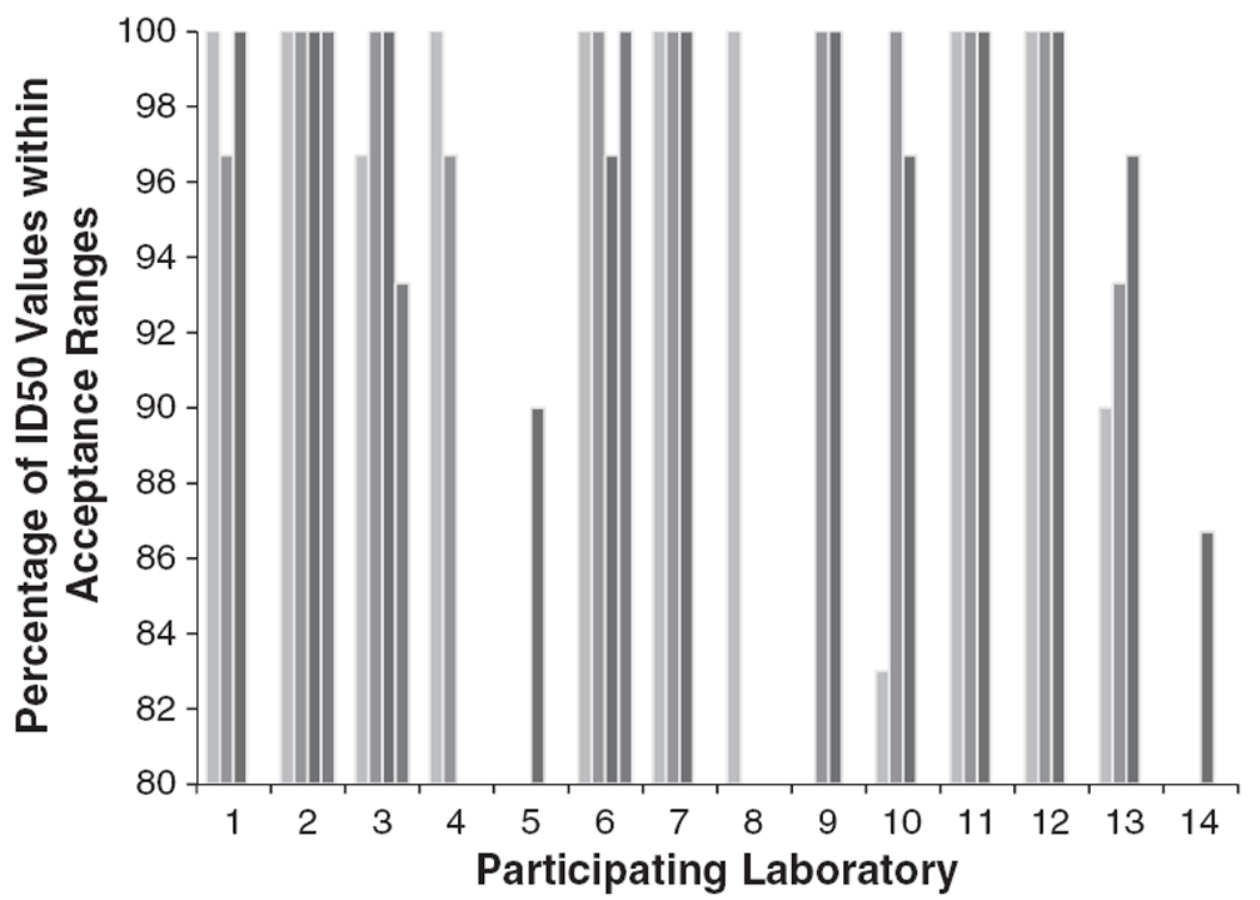

- PT Round 1 - PT Round 2

- PT Round 3 - PT Round 4

Fig. 4.

Laboratory performance in the standardized proficiency testing program. Bars represent the percentage of ID50 values within the acceptance ranges from each participating laboratory over multiple rounds of proficiency testing (PT Rounds 1-4). 


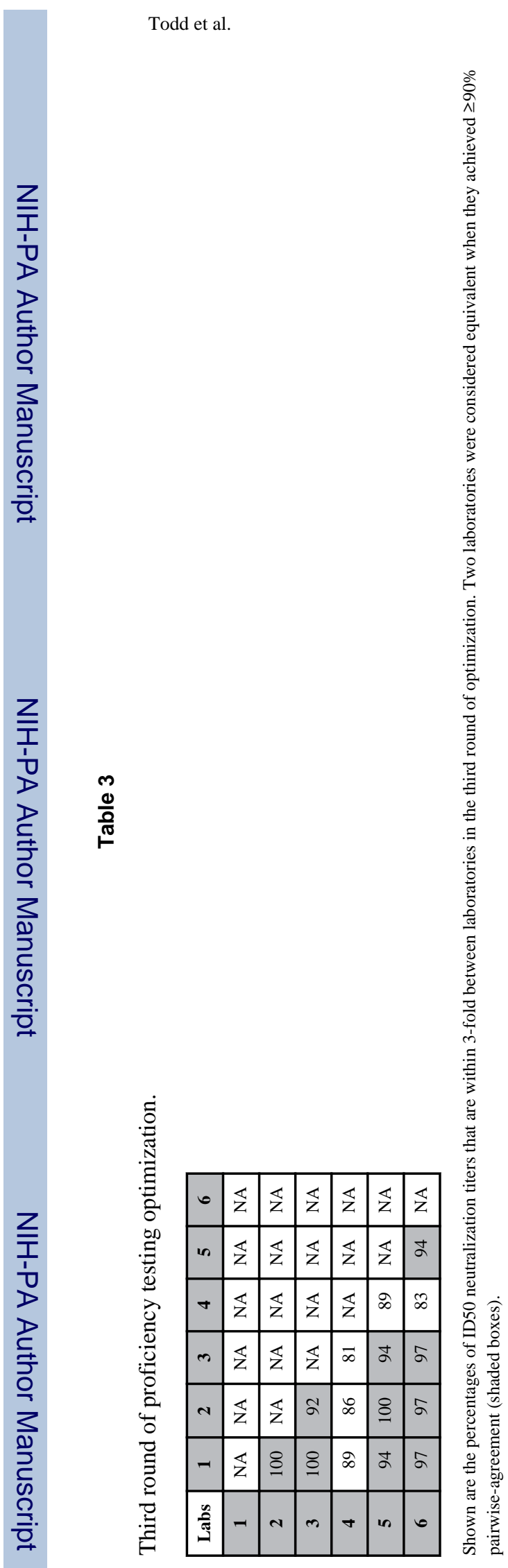

J Immunol Methods. Author manuscript; available in PMC 2013 January 31. 


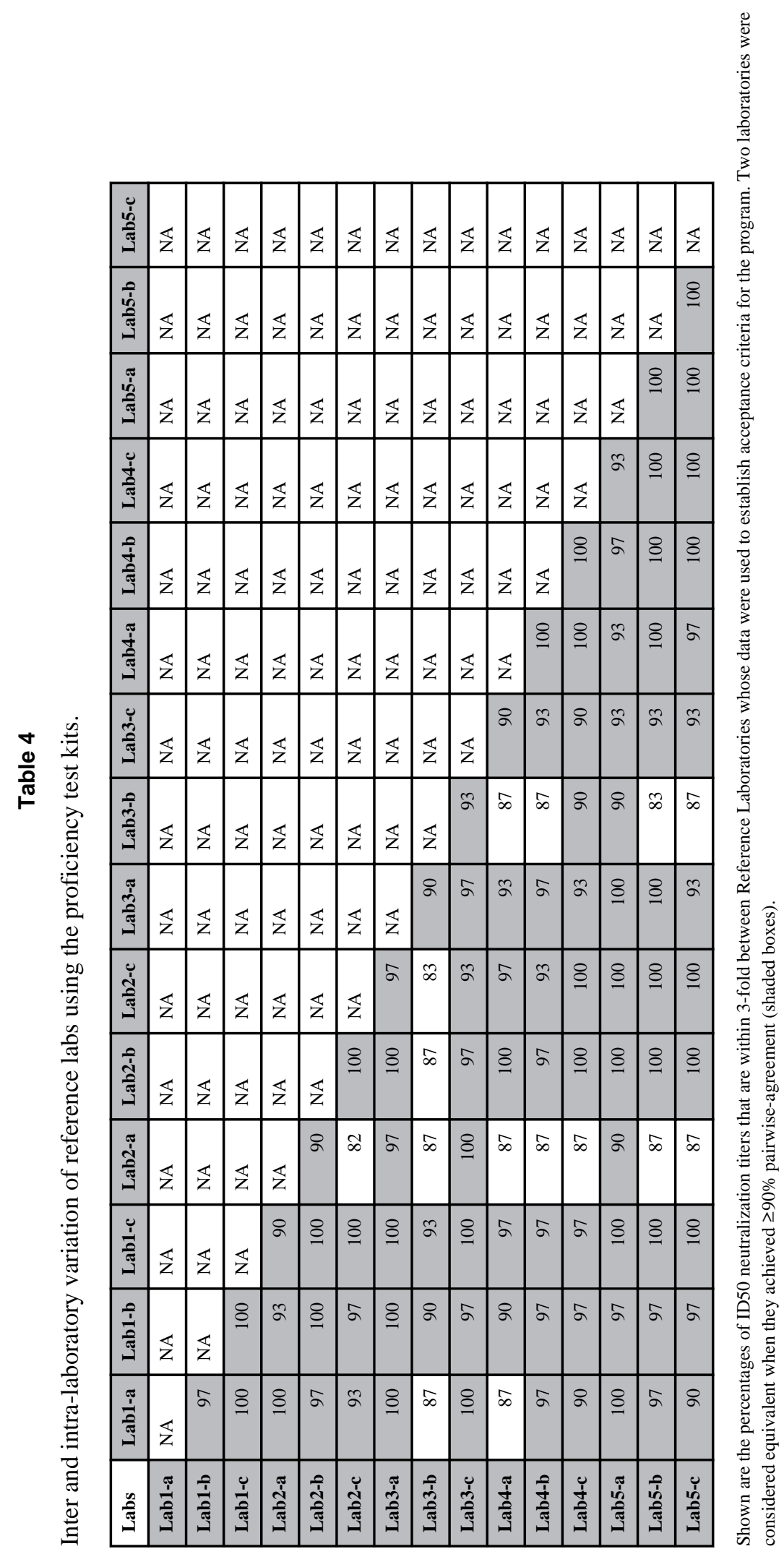




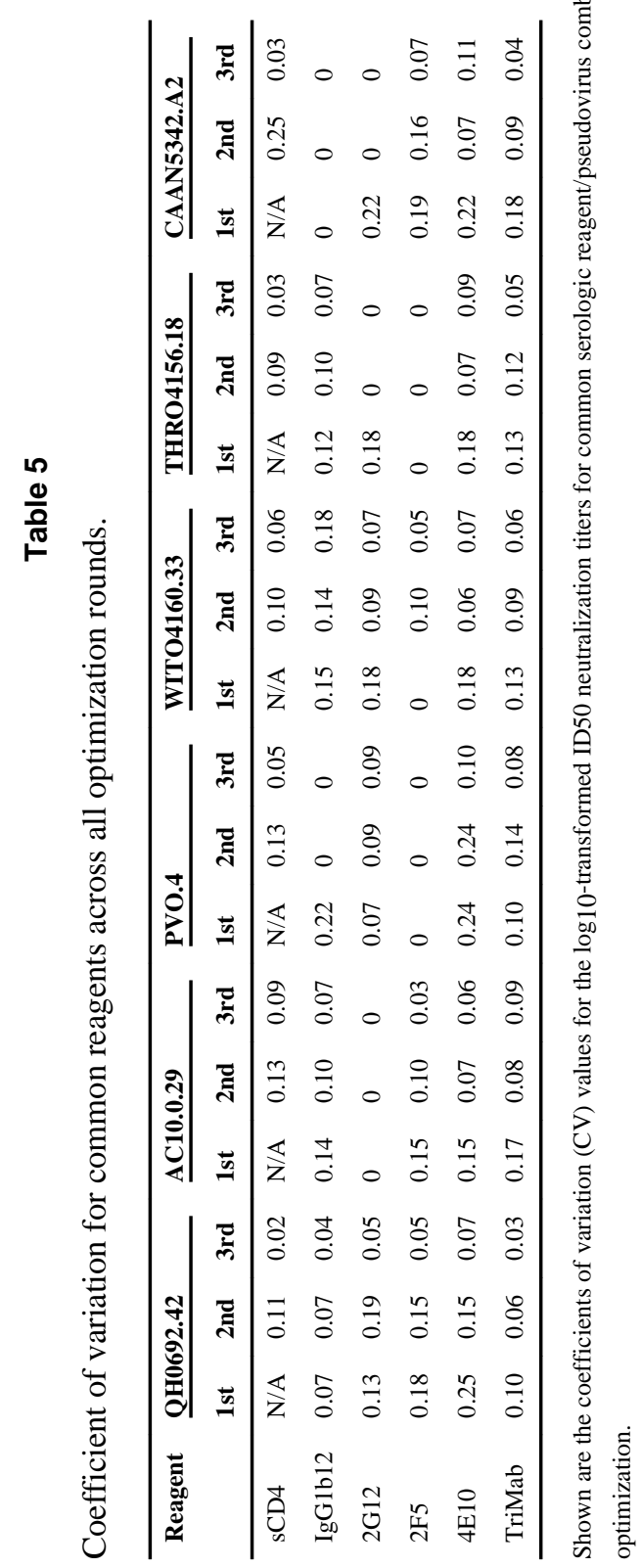

